\title{
Colliding Message Pair for 53-Step HAS-160
}

\author{
Florian Mendel ${ }^{\star}$ and Vincent Rijmen \\ Institute for Applied Information Processing and Communications (IAIK), \\ Graz University of Technology, Inffeldgasse 16a, A-8010 Graz, Austria
}

\{Florian.Mendel, Vincent.Rijmen\}@iaik.tugraz.at

\begin{abstract}
HAS-160 is an iterated cryptographic hash function that is widely used in Korea. In this article, we present a collision attack on the hash function HAS-160 reduced to 53-steps. The attack has a complexity of about $2^{35}$ hash computations. It is based on the work of Cho et al. presented at ICISC 2006. We improve the attack complexity of Cho et al. by a factor of about $2^{20}$ using a slightly different strategy for message modification in the first 20 steps of the hash function and present the first actual colliding message pair for 53-step HAS-160. Furthermore, we show how the attack can be extended to 59-step HAS-160 by using a characteristic spanning over two message blocks.
\end{abstract}

\section{Introduction}

HAS-160 is an iterated cryptographic hash function that is widely used in Korea and standardized by Korean government (TTAS.KO-12.0011/R1) [1]. It is an iterated cryptographic hash function that produces a 160-bit hash value. The design of HAS-160 is based on design principles of the MD4 family. Recently, weaknesses have been shown for several members of the MD4 family such as MD5 [5] and SHA-1 [6]. These breakthrough results by Wang et al. in the cryptanalysis of hash functions, are the motivation for intensive research in the design and analysis of hash functions. In 4, Yun et al. applied the techniques invented by Wang et al. in the cryptanalysis of MD5 and SHA-1 to the HAS-160 hash function. In their article they show that a collision can be found for HAS-160 reduced to 45 (out of 80 ) steps with a complexity of about $2^{12} 45$-step HAS-160 computations. This attack was later extended by Cho et al. to HAS-160 reduced to 53 steps. Their attack has a complexity of about $2^{55} 53$-step HAS-160 computations. This is not feasible on an ordinary PC in practice.

In this article, we show how to improve their attack by using a slightly different message modification technique to fulfill the conditions on the state variables in the first 20 steps of the hash function. With our method, we find a colliding message pair for 53-step HAS-160 with a complexity of about $2^{35}$ hash computations. This improves the attack complexity of the original attack of Cho et al. by a factor of $2^{20}$, which makes the attack feasible in practice.

\footnotetext{
* This author is supported by the Austrian Science Fund (FWF), project P18138.
} 
Furthermore, we show how the attack can be extended to 59 steps of HAS-160. The attack has a complexity of about $2^{55}$ hash computations.

The remainder of this article is structured as follows. A description of the hash function is given in Section 2. The collision attack of Cho et al. is described in Section 3. In Section 4, we describe the new improved collision attack. A sample colliding message pair is given in Section 5. Section 6 shows how the attack can be extended to 59 steps of HAS-160 by using a characteristic spanning over two message blocks. Finally, conclusions are presented in Section 7 .

\section{Description of the HAS-160}

HAS-160 is an iterative hash function that processes 512-bit input message blocks and produces a 160-bit hash value. The design of HAS-160 is similar to the design principles of MD5 and SHA-1. In the following, we briefly describe the hash function. It basically consists of two parts: message expansion and state update transformation. A detailed description of the HAS-160 hash function is given in 1]. Throughout the remainder of this article, we will follow the notation given in Table 1.

Table 1. Notation

\begin{tabular}{|c|l|}
\hline Notation & Meaning \\
\hline$A \vee B$ & logical OR of two bit-strings $A$ and $B$ \\
$A \wedge B$ & logical AND of two bit-strings $A$ and $B$ \\
$A \oplus B$ & logical XOR of two bit-strings $A$ and $B$ \\
$A \lll n$ & bit-rotation of $A$ by $n$ positions to the left \\
$M_{j}$ & message block $j$ (512-bits) \\
$m_{i}$ & message word $i$ (32-bits) \\
$w_{i}$ & expanded message word $i$ (32-bits) \\
\hline
\end{tabular}

Message Expansion. The message expansion of HAS-160 is a permutation of 20 expanded message words $w_{i}$ in each round. The 20 expanded message words $w_{i}$ used in each round are constructed from the 16 input message words $m_{i}$ as follows.

\begin{tabular}{|c|c|c|c|c|}
\hline & Round 1 & Round 2 & Round 3 & Round 4 \\
\hline$w_{0}$ & $m_{0}$ & $m_{0}$ & $m_{0}$ & $m_{0}$ \\
$\vdots$ & $\vdots$ & $\vdots$ & $\vdots$ & $m_{15}$ \\
$w_{15}$ & $m_{15}$ & $m_{15}$ & $m_{15}$ & $m_{5}$ \\
\hline$w_{16}$ & $w_{0} \oplus w_{1} \oplus w_{2} \oplus w_{3}$ & $w_{3} \oplus w_{6} \oplus w_{9} \oplus w_{12}$ & $w_{12} \oplus w_{5} \oplus w_{14} \oplus w_{7}$ & $w_{7} \oplus w_{2} \oplus w_{13} \oplus w_{8}$ \\
\hline$w_{17}$ & $w_{4} \oplus w_{5} \oplus w_{6} \oplus w_{7}$ & $w_{15} \oplus w_{2} \oplus w_{5} \oplus w_{8}$ & $w_{0} \oplus w_{9} \oplus w_{2} \oplus w_{11}$ & $w_{3} \oplus w_{14} \oplus w_{9} \oplus w_{4}$ \\
\hline$w_{18}$ & $w_{8} \oplus w_{9} \oplus w_{10} \oplus w_{11}$ & $w_{11} \oplus w_{14} \oplus w_{1} \oplus w_{4}$ & $w_{4} \oplus w_{13} \oplus w_{6} \oplus w_{15}$ & $w_{15} \oplus w_{10} \oplus w_{5} \oplus w_{0}$ \\
\hline$w_{19}$ & $w_{12} \oplus w_{13} \oplus w_{14} \oplus w_{15}$ & $w_{7} \oplus w_{10} \oplus w_{13} \oplus w_{0}$ & $w_{8} \oplus w_{1} \oplus w_{10} \oplus w_{3}$ & $w_{11} \oplus w_{6} \oplus w_{1} \oplus w_{12}$ \\
\hline
\end{tabular}


For the ordering of the expanded message words $w_{i}$ the following permutation is used:

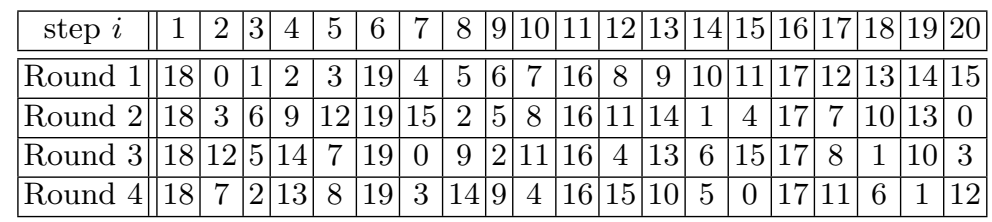

State Update Transformation. The state update transformation of HAS-160 starts from a (fixed) initial value $I V$ of five 32-bit registers and updates them in 4 rounds of 20 steps each. Figure 1 shows one step of the state update transformation of the hash function.

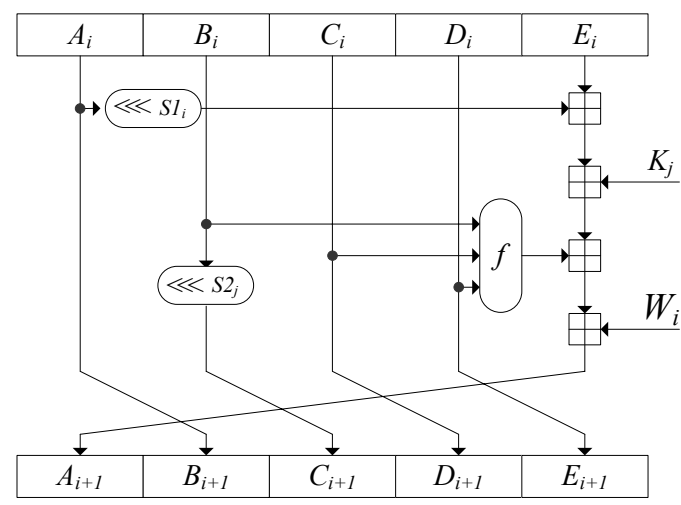

Fig. 1. The step function of HAS-160.

Note that the function $f$ is different in each round: $f_{0}$ is used in the first round, $f_{1}$ is used in round 2 and round 4 , and $f_{2}$ is used in round 3.

$$
\begin{aligned}
& f_{0}(x, y, z)=(x \wedge y) \oplus(\neg x \wedge z) \\
& f_{1}(x, y, z)=x \oplus y \oplus z \\
& f_{2}(x, y, z)=(x \vee \neg z) \oplus y
\end{aligned}
$$

A step constant $K_{j}$ is added in every step; the constant is different for each round. For the actual values of the constants we refer to [1]. While rotation value $s_{2} \in\{10,17,25,30\}$ is different in each round of the hash function, the rotation value $s_{1}$ is different in each step of a round. The rotation value $s_{1}$ for each step of a round is given below. 


\begin{tabular}{|c|c|c|c|c|c|c|c|c|c|c|c|c|c|c|c|c|c|c|c|c|}
\hline step $i$ & 1 & 2 & 3 & 4 & 5 & 6 & 7 & 8 & 9 & 10 & 11 & 12 & 13 & 14 & 15 & 16 & 17 & 18 & 19 & 20 \\
\hline \hline$s_{1}$ & 5 & 11 & 7 & 15 & 6 & 13 & 8 & 14 & 7 & 12 & 9 & 11 & 8 & 15 & 6 & 12 & 9 & 14 & 5 & 13 \\
\hline
\end{tabular}

After the last step of the state update transformation, the initial value and the output values of the last step are combined, resulting in the final value of one iteration known as Davies-Meyer hash construction (feed forward). In detail, the feed forward is a word-wise modular addition of the $I V$ and the output of the state update transformation. The result is the final hash value or the initial value for the next message block.

\section{The Attack on 53-step HAS-160}

In this section, we will briefly describe the attack of Cho et al. on 53-step HAS-160. A detailed description of the attack is given in 3 . For a good understanding of our results it is recommended to study it carefully.

The attack is based on recent results in cryptanalysis of hash functions [5]6]. It can be basically described as follows.

1. Find a characteristic for the hash function that holds with high probability after the first round of the hash function.

2. Find a characteristic (not necessarily with high probability) for the first round of the hash function.

3. Use message modification techniques [5] to fulfill conditions for the characteristic in the first round. This increases the probability of the characteristic.

4. Use random trials to find values for the message bits such that the message follows the characteristic.

\subsection{Characteristic for 53-step HAS-160}

Finding a good characteristic was the most difficult part of the attack. In Table2, the characteristic which is used by Cho et al. in the collision attack on 53-step HAS-160 is given. Note that we use the same differential path for our improved collision-attack in Section 4 .

Signed-bit differences [5] are used to describe the differential path for 53-step HAS-160. To improve readability, only the differences in the expanded message words and state variable $A$ for each step are given. Note that the differences of the state variables $B, C, D, E$ are defined by the differences in state variable $A$.

\subsection{Set of Sufficient Conditions}

In order to guarantee that the message follows the characteristic given in Table 2 . a set of conditions on the state variables has to be fulfilled. In Table 3 , the set of sufficient conditions for the first 25 steps of the hash function is given. 
Table 2. Characteristic for 53-step HAS-160 (cf. [3]).

\begin{tabular}{|c|c|c|}
\hline step & $\Delta A$ & $\Delta w$ \\
\hline 1 & -32 & 32 \\
\hline 2 & $11,-12$ & $\cdot$ \\
\hline 3 & $18, \ldots, 21,-22$ & $\cdot$ \\
\hline 4 & $1, \ldots, 16,-17$ & $\cdot$ \\
\hline 5 & $7,8,-9,18,-19,32$ & 32 \\
\hline 6 & $3,-4,17,-20,-21,22$ & 32 \\
\hline 7 & $-14, \ldots,-17,18,22,29$ & $\cdot$ \\
\hline 8 & $4,-10,11$ & $\cdot$ \\
\hline 9 & $13,-15,16,18,-19,30,31,-32$ & 32 \\
\hline 10 & $-10,-11,12,-17,-24,25$ & $\cdot$ \\
\hline 11 & $-1,2,-13, \ldots,-15,16,28,-32$ & 32 \\
\hline 12 & $-8,9,-17,-21,22,26$ & 32 \\
\hline 13 & $8,10,11,-12,26,27,-28$ & $\cdot$ \\
\hline 14 & $-10,17,18,-19,28, \ldots, 30,-31$ & $\cdot$ \\
\hline 15 & $11, \ldots, 14,-15,-16,20,-23,26,-27$ & $\cdot$ \\
\hline 16 & $-1,5,6,-7,-11,-12,13,-20,21,-22,-31,32$ & 32 \\
\hline 17 & $-11,18,-20,-26,27$ & $\cdot$ \\
\hline 18 & $1,5,18,20,22,27$ & $\cdot$ \\
\hline 19 & $4,13,30$ & $\cdot$ \\
\hline 20 & $-4,11$ & 32 \\
\hline 21 & 11 & $\cdot$ \\
\hline 22 & -30 & 32 \\
\hline 23 & $\cdot$ & 32 \\
\hline 24 & 15 & $\cdot$ \\
\hline 25 & -15 & $\cdot$ \\
\hline 26 & $\cdot$ & $\cdot$ \\
\hline 27 & $\cdot$ & 32 \\
\hline 28 & $\cdot$ & $\cdot$ \\
\hline 29 & $\cdot$ & $\cdot$ \\
\hline 30 & $\cdot$ & $\cdot$ \\
\hline 31 & $\cdot$ & $\cdot$ \\
\hline$\vdots$ & $\cdot$ & $\cdot$ \\
\hline 53 & & \\
\hline
\end{tabular}


Table 3. A set of sufficient conditions on $A_{i}$ for the differential path given in Table 2, where 'a' denotes a condition $A_{i, j}=A_{i-1, j}$, 'b' denotes a condition $A_{i, j} \neq A_{i-1, j}$, 'c' denotes a condition $A_{i, j} \neq A_{i-1, j+7}$, 'd' denotes a condition $A_{i, j}=A_{i-1, j-10}$, 'e' denotes a condition $A_{i, j}=A_{i-1, j-17}$, and 'f' denotes a condition $A_{i, j} \neq A_{i-1, j-17}$.

\begin{tabular}{|c|c|c|c|c|}
\hline \multirow{2}{*}{ state variable } & \multicolumn{3}{|c|}{ condition on bits } & \multirow{2}{*}{ \#conditions } \\
\hline & $32-25$ & $24-17$ & $16-9$ & \\
\hline$A_{1}$ & $1------$ & -------- & $----110-1-----a a$ & 7 \\
\hline$A_{2}$ & $0100---1$ & ------- & ----100 a $01---1--$ & 12 \\
\hline$A_{3}$ & $1100 a a a 0$ & aa10000- - & ----10 10aaa0aa & 25 \\
\hline$A_{4}$ & $11000-11$ & $--10---1$ & 0000000000000000 & 26 \\
\hline$A_{5}$ & 01110111 & 00110100 & $0010011100-11---$ & 28 \\
\hline$A_{6}$ & $0--00111$ & $110111-0$ & $0000001-00 a 010-0$ & 27 \\
\hline$A_{7}$ & $1010101-$ & $--0-1001$ & $1111--10-------1$ & 19 \\
\hline$A_{8}$ & $100-0000$ & $1-1 \mathrm{a} 0---$ & $-111-011$ a0aa0a11 & 25 \\
\hline$A_{9}$ & $100-0101$ & $10-0010-$ & $0110-----11-0-00$ & 22 \\
\hline$A_{10}$ & $--000-10$ & $10 \mathrm{a} 01-01$ & $1-1-0110000010--$ & 24 \\
\hline$A_{11}$ & $1 \mathrm{a} 100010$ & $-1001-10$ & $0111000101---001$ & 27 \\
\hline$A_{12}$ & $1-1--100$ & 10011111 & $1--00001-0--110$ & 23 \\
\hline$A_{13}$ & $00--1001$ & $11-00000$ & $1---1001$ 0a110-11 & 25 \\
\hline$A_{14}$ & $010001-0$ & --101100 & $0--\mathrm{a} 101---111 \mathrm{a} 00$ & 24 \\
\hline$A_{15}$ & ---11101 & $-1000-10$ & $1100000101100 \mathrm{a} 00$ & 27 \\
\hline$A_{16}$ & 01010111 & $00101--01$ & $1--01110 \quad 010010-1$ & 27 \\
\hline$A_{17}$ & $111--011$ & $01011-00$ & $00-10110 \quad 0--10--1$ & 24 \\
\hline$A_{18}$ & $01--00--$ & $-00-0-001$ & $11-1--11---00 b-0$ & 18 \\
\hline$A_{19}$ & $--0-----$ & $---d-c--$ & $-0-0-1------0---$ & 7 \\
\hline$A_{20}$ & $----0-b-$ & $---\mathrm{b}-----$ & $-----0-----f 1---$ & 6 \\
\hline$A_{21}$ & $--f-0---$ & -------- & $-f-a-0----------$ & 5 \\
\hline$A_{22}$ & $--1-----$ & $---\mathrm{e}-----$ & $---------\quad---------$ & 2 \\
\hline$A_{23}$ & $----f---$ & -------- & $-\mathrm{e}^{------}--------$ & 2 \\
\hline$A_{24}$ & $--\mathrm{b}------$ & -------- & $-0--------------$ & 2 \\
\hline$A_{25}$ & -------- & -------- & $-1--------------$ & 1 \\
\hline
\end{tabular}




\subsection{Finding a Colliding Message Pair}

In order to find a colliding message pair, we have to find a message that fulfills all the conditions given in Table 3. In total there are 434 conditions on the state variables. Therefore, the probability that a random message follows the characteristic can be estimated by $2^{-434}$.

However, the probability can be improved by using message modification techniques. The main idea of message modification is to use the degrees of freedom one has in the choice of the message words to fulfill conditions on the state variables. This improves the probability of the characteristic. It is clear that the number of conditions that can not be fulfilled by message modification techniques determine the final attack complexity.

In [3], Cho et al. describe an algorithm for finding a colliding message pair for 53 -step HAS-160. The algorithm has a complexity of about $2^{55} 53$-step HAS-160 computations. It can be summarized as follows:

1. Use basic message modification techniques to fulfill all conditions on state variables $A_{1}, \ldots, A_{10}$. This determines the message words $m_{0}, m_{1}, m_{2}, m_{3}$, $m_{4}, m_{5}, m_{6}, m_{7}$ and the values for $m_{8} \oplus m_{9} \oplus m_{10} \oplus m_{11}$ and $m_{12} \oplus m_{13} \oplus m_{14} \oplus$ $m_{15}$. This adds only small additional cost to the attack complexity. Only 10 steps of HAS-160 have to be computed to determine all these message words.

2. At step 11, the dependent expanded message word $w_{16}=m_{0} \oplus m_{1} \oplus m_{2} \oplus m_{3}$ is already fixed by the first step of the attack. Since there are 27 conditions on $A_{11}$ in step 11, we may fulfill them with a probability of $2^{-27}$. Hence, we have to repeat step 1 of the attack about $2^{27}$ times to find message words satisfying all conditions in steps 1-11.

Finishing this step of the attack has a complexity of about $2^{27} \cdot 11$ step computations of HAS-160.

3. Use again basic message modification techniques to fulfill all conditions on $A_{12}, A_{13}, A_{14}$. This determines the message words $m_{8}, m_{9}, m_{10}$. Since these message words can be chosen freely, this adds only small additional cost (3 step computations of HAS-160) to the attack complexity.

4. At step 15 and 16, the dependent words $m_{11}$ and $m_{4} \oplus m_{5} \oplus m_{6} \oplus m_{7}$ are already fixed by the previous steps of the attack. Since there are $27+27=54$ conditions on $A_{15}$ and $A_{16}$, we may fulfill these conditions with a probability of $2^{-54}$. Since there are about $2^{24}$ possible choices for $m_{8}, m_{9}, m_{10}$ in the third step of the attack (see Table 3), step 3 of the attack can only be repeated $2^{24}$ times. Hence, the whole attack has to be repeated about $2^{30}$ times to find message words following the characteristic in steps 1-16.

Finishing this step of the attack has a complexity of about $2^{30} \cdot\left(2^{27} \cdot 11+2^{24} \cdot 5\right)$ step computations of HAS-160. This is approximately about $2^{55} 53$-step HAS-160 computations.

5. To fulfill the conditions on $A_{17}, A_{18}, A_{19}$ basic message modification techniques are used again. This determines the message words $m_{12}, m_{13}, m_{14}$. Since these values can be chosen freely, this adds only small additional cost (3 step computations of HAS-160) to the attack complexity. 
6. After step 19, all the message words have been determined. Since there are still 18 conditions on $A_{20}, \ldots, A_{25}$ (see Table 3 ), we can satisfy them with a probability of $2^{-18}$. Therefore, step 5 of the attack has to be repeated about $2^{18}$ times to fulfill all the conditions in steps 20-25. This adds negligible cost to the final attack complexity.

With this method a collision can be found in 53-step HAS-160 with a complexity of about $2^{55} 53$-step HAS-160 computations. For a detailed description of the attack we refer to [3].

\section{Improved Collision Attack}

In this section, we show how the attack complexity can be reduced to $2^{35}$. In the attack, we use the differential path of Cho et al. given in Section 3 To improve the attack complexity, we use a slightly modified strategy for message modification in the first 16 steps of the hash function. The main idea of our new method is to reduce the complexity of the collision search algorithm in steps 1-16. Therefore, we use the fact that an additional (first) message block can be used to generate an arbitrary $I V$ (for the second block). This additional degree of freedom we use to reduce the complexity of the attack. The attack can be summarized as follows.

1. Choose arbitrary values for $A_{2}, A_{3}, A_{4}, A_{5}, A_{6}$ satisfying all conditions.

2. Apply message modification techniques to steps $7-15$. This determines the message words $m_{4}, m_{5}, m_{6}, m_{7}, m_{8}, m_{9}, m_{10}, m_{11}$ and the value for $m_{0} \oplus m_{1} \oplus$ $m_{2} \oplus m_{3}$. At step 16, the dependent words $m_{4}, m_{5}, m_{6}, m_{7}$ are already used. Since there are 27 conditions at that step (see Table 3), we may satisfy all the conditions from step 7 up to step 16 with a probability of $2^{-27}$. Hence, we have to compute about $2^{27} \cdot 10$ steps of HAS-160 to find message words that follow the characteristic from step 7 to 16 .

3. Repeat step 2 of the attack about $2^{7}$ times to get about $2^{7}$ different values for $m_{8} \oplus m_{9} \oplus m_{10} \oplus m_{11}$ and save them in a list $L$. We will need these values in the next step of the attack.

Finishing this step of the attack has a complexity of about $2^{7} \cdot 2^{27} \cdot 10$ step computations of HAS- 160 . This is equivalent to about $2^{31.6} 53$-step HAS-160 computations.

4. Use an arbitrary (first) message block to get a suitable $I V$ (for the second block). Note that we have to calculate on average $2 I V \mathrm{~s}$, since 1 condition on the $I V$ has to be fulfilled to guarantee that the characteristic holds in the following steps.

Calculate $A_{1}$ (for all values in $L$ ) and check if the 7 conditions on $A_{1}$ are satisfied. Since there are 7 conditions on $A_{1}$, we always expect to meet the conditions after trying all $2^{7}$ values in the list $L$.

Once, we have determined $A_{1}$, this also determines $m_{0}, m_{1}, m_{2}, m_{3}$ and $m_{12} \oplus$ $m_{13} \oplus m_{14} \oplus m_{15}$. Since $m_{0} \oplus m_{1} \oplus m_{2} \oplus m_{3}$ has already been fixed in the 
second step of the attack, this step of the attack succeeds with probability $2^{-32}$.

Hence, we have to repeat this step of the attack about $2^{32}$ times to find message words following the characteristic in steps 1-16.

Finishing this step of the attack has a complexity of about $2^{32} \cdot\left(2^{7}+5+53 \cdot 2\right)$ step computations of HAS-160. This is approximately $2^{34.2} 53$-step HAS-160 computations.

5. Do steps 17 to 25 as described in the original attack (see Section 3). The complexity of these steps can be neglected for the final attack complexity.

Hence, we can find a colliding message pair for 53-step HAS-160 with a complexity of about $2^{31.6}+2^{34.2} \approx 2^{35} 53$-step HAS-160 computations. Note that the complexity of the attack can be slightly improved by increasing the size of the list $L$. A colliding message pair for 53-step HAS-160 is given in the next section.

\section{A Colliding Message for 53-step HAS-160}

With our improved collision attack, we can construct a collision with a complexity of about $2^{35} 53$-step HAS-160 computations. The colliding message pair is given in Table 4. Note that $h_{0}$ is the initial value, $h_{1}$ is the intermediate hash value after the first block, and $h_{2}$ is the final hash value after the second block.

Table 4. A colliding message pair for HAS-160.

\begin{tabular}{|c|c|c|c|c|c|c|c|}
\hline$h_{0}$ & 67452301 EFCDAB89 & 98BADCFE & 10325476 & C3D2E1F0 & & & \\
\hline \multirow{2}{*}{$M_{0}$} & 34338ECF ED111A03 & EB2EE891 & 763594E3 & 96080160 & 4558A929 & EC731044 & B7BADD0B \\
\hline & BC637C76 B21FA220 & 47493D4D & B2AEAB79 & $\mathrm{A} 68354 \mathrm{CF}$ & 227 & 46DE18D7 & $5 \mathrm{~F} 3 \mathrm{~B}$ \\
\hline$h_{1}$ & 40D4B34F F1185C20 & ADE02611 & 9B666A7E & 34769338 & & & \\
\hline \multirow{2}{*}{$M_{1}$} & 4E8F4717 D8E79F84 & 89D8FE81 & 04B34CA7 & 01EA3C40 & A364A502 & 059F6AB9 & 22774031 \\
\hline & 9F3E80CE D647A926 & $1 \mathrm{~F} 61242 \mathrm{~A}$ & $\mathrm{~A} 1 \mathrm{E} 224 \mathrm{AB}$ & 901A5AEE & 1BCEEEB1 & $\mathrm{EDE}$ & F9A \\
\hline \multirow{2}{*}{$M_{1}^{\prime}$} & 4E8F4717 D8E79F84 & 89D8FE81 & 84B34CA7 & 01EA3C40 & A364A502 & 859F6AB9 & 22774031 \\
\hline & 1F3E80CE D647A926 & $1 \mathrm{~F} 61242 \mathrm{~A}$ & $\mathrm{~A} 1 \mathrm{E} 224 \mathrm{AB}$ & 901A5AEE & 1BCEEEB1 & EDEAA891 & DFF9A \\
\hline \multirow{2}{*}{$\Delta M_{1}$} & 00000000 & 00000 & 80000000 & 00000000 & 000 & 800 & 00000000 \\
\hline & 8000000000000000 & 00000000 & 00000000 & 00000000 & 00000000 & 00000000 & 80000000 \\
\hline$h_{2}$ & DA815BDF & DF265AB5 & 819CDE2E & B887F3E & & & \\
\hline$h_{2}^{\prime}$ & 96D30020 DA815BDF & DF265AB5 & 819CDE2E & 5B887F3E & & & \\
\hline
\end{tabular}

\section{Extending the attack to 59 steps of HAS-160}

In this section, we show how the attack of Cho et al. on 53-step HAS-160 can be extended to 59 steps. In [5], Wang et al. show a collision attack for MD5 spanning over two message blocks. In the attack, they use a second message block to turn a near-collision after the first message block into a collision after feed forward of the second message block. Hence, two related near-collisions can be used to produce a two-block collision for the hash function. Therefore, a 


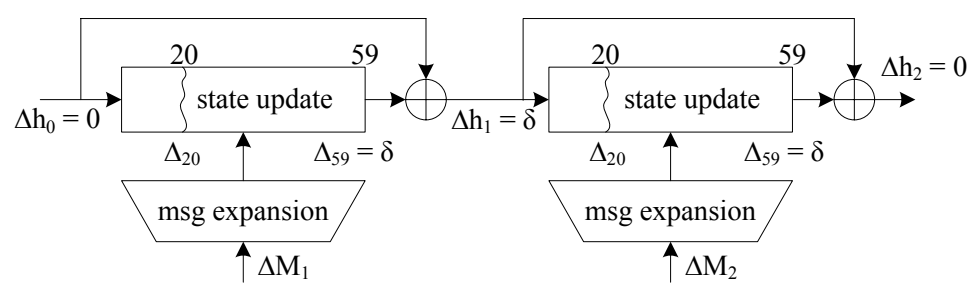

Fig. 2. A two-block collision in the hash function.

different characteristic is required in the first round of the second block. This is depicted in Fig. 2 .

For the attack on 59-step HAS-160, we use the characteristic given in Table5. Note that the characteristic from step 20 to step 53 is equal to the characteristic used by Cho et al. in the attack on 53-step HAS-160. Hence, we can use the same characteristic as Cho et al. in the first 20 steps for the first message block. Since the characteristic has a probability of $2^{-32}$ to hold in steps 54 to 59 , we have an attack complexity for the first message block of about $2^{50}$ (respectively $2^{55}$ ) hash computations following the attack strategy described in Section 4 (respectively Section 3p.

Table 5. Near-collision producing characteristic for 59-step HAS-160.

\begin{tabular}{|c|c|c|}
\hline step & $\Delta A$ & $\Delta w$ \\
\hline 20 & $-4,11$ & 32 \\
\hline 21 & 11 & $\cdot$ \\
\hline 22 & -30 & 32 \\
\hline 23 & $\cdot$ & 32 \\
\hline 24 & 15 & $\cdot$ \\
\hline 25 & -15 & $\cdot$ \\
\hline 26 & $\cdot$ & $\cdot$ \\
\hline 27 & $\cdot$ & 32 \\
\hline 28 & $\cdot$ & $\cdot$ \\
\hline 29 & $\cdot$ & $\cdot$ \\
\hline 30 & $\cdot$ & 32 \\
\hline 31 & $\cdot$ & $\cdot$ \\
\hline$\vdots$ & $\vdots$ & $\vdots$ \\
\hline 53 & $\cdot$ & $\cdot$ \\
\hline 54 & 32 & 32 \\
\hline 55 & 6,32 & 32 \\
\hline 56 & 12,18 & $\cdot$ \\
\hline 57 & $21,-25,27$ & 32 \\
\hline 58 & $3,-7,9,31$ & $\cdot$ \\
\hline 59 & $-4,8,11,13$ & $\cdot$ \\
\hline
\end{tabular}


However, for the second message block a slightly different characteristic is needed for the first 20 steps of the hash function. In the analysis, we assume that such a characteristic can be found (once the first message block has been fixed) like it was done for 64-step SHA-1 [2. Furthermore, it seems to be reasonable to assume that this characteristic has roughly the same probability as the characteristic used in the first message block. Thus, the complexity of the second block is about $2^{55}$ hash computations. This results in a complexity of about $2^{50}+2^{55} \approx 2^{55}$ hash computations to construct a two-block collision for HAS-160 reduced to 59 steps.

\section{Conclusion}

In this article, we presented an improved collision attack on 53-step HAS-160 and an actual colliding message pair. Our new improved attack has a complexity of about $2^{35} 53$-step HAS-160 computations. In the attack, we used a slightly modified strategy to do message modification in HAS-160. With this method, we can improve the previous attack by a factor of $2^{20}$, which makes the attack feasible in practice. Furthermore, we show how the attack can be extended to 59 steps of HAS-160 by using a characteristic spanning over two message blocks. The attack has a complexity of about $2^{55}$ hash computations. However, whether or not the attack of Cho et al. can be extended to the full HAS-160 hash function remains a topic of further research.

\section{Acknowledgment}

The authors would like to thank Aaram Yun for fruitful discussions on this article.

\section{References}

1. Telecommunications Technology Association. Hash Function Standard Part 2: Hash Function Algorithm Standard (HAS-160), TTAS.KO-12.0011/R1, December 2000.

2. Christophe De Cannière and Christian Rechberger. Finding SHA-1 Characteristics: General Results and Applications. In Xuejia Lai and Kefei Chen, editors, ASIACRYPT, volume 4284 of LNCS, pages 1-20. Springer, 2006.

3. Hong-Su Cho, Sangwoo Park, Soo Hak Sung, and Aaram Yun. Collision Search Attack for 53-Step HAS-160. In Min Surp Rhee and Byoungcheon Lee, editors, ICISC, volume 4296 of LNCS, pages 286-295. Springer, 2006.

4. Aaram Yun, Soo Hak Sung, Sangwoo Park, Donghoon Chang, Seokhie Hong, and Hong-Su Cho. Finding Collision on 45-step HAS-160. In Dongho Won and Seungjoo Kim, editors, ICISC, volume 3935 of LNCS, pages 146-155. Springer, 2005.

5. Xiaoyun Wang and Hongbo Yu. How to Break MD5 and Other Hash Functions. In Ronald Cramer, editor, EUROCRYPT, volume 3494 of LNCS, pages 19-35. Springer, 2005.

6. Xiaoyun Wang, Yiqun Lisa Yin, and Hongbo Yu. Finding Collisions in the Full SHA-1. In Victor Shoup, editor, CRYPTO, volume 3621 of LNCS, pages 17-36. Springer, 2005. 